\title{
Multifractality of Aeroengine Fuel Flow Time Series
}

\section{Hong Zhang ${ }^{1}$, and Keqiang Dong ${ }^{2, \text { a }}$}

\author{
${ }^{1}$ Basic Courses Department, Tianjin Sino-German University of Applied Sciences, Tianjin 300350, \\ China; \\ ${ }^{2}$ School of Science, Civil Aviation University of China, Tianjin 300300, China. \\ ahongzhangdong@163.com
}

\begin{abstract}
In this paper, we analyze the data of aeroengine performance parameters fuel flow (FF). Using the Box-counting dimension method, we find that the aeroengine performance parameters have different power law exponents in the plot for the number of box and diameter of box, which indicates the multifractality exist in the time series. In order to investigate the latent properties of the data, the generalized fractal dimension are calculated. The results show that the multifractality exist in the aeroengine performance data.
\end{abstract}

Keywords: Fractal; Aeroengine time series; Time series; Fuel flow

\section{基于燃油流量序列的航空发动机分形特征分析}

张宏 ${ }^{1}$, 董科强 ${ }^{2}$

（1. 天津中德应用技术大学 基础课部, 天津 中国; 2. 中国民航大学 理学院, 天津 中国)

摘要: 本文应用分形测量方法, 分析了发动机性能参数, 发现燃油流量时间序列的配分函数具有弱非线性特征。 进一步借助广义分形维数对燃油流量时间序列进行研究, 结果表明, 燃油流量时间序列具有一定的多重分形特性。 这将为多重分形在发动机性能参数的非线性研究提供重要的理论基础。

关键词:广义分形维数; 时间序列; 航空发动机; 燃油流量

\section{1 前言}

随着航空业的迅速发展, 为飞行器提供推进动力的航空发动机, 得到了迅猛发展。发动机作为飞行器 的心脏, 对其进行健康监控并对其进行状态估和故障诊断, 对于推动视情维修、减少飞机运与维修成本、 提高飞机安全性具有重要的意义 $[1,2]$ 。在巡航状态下, 发动机性能参数的变化趋势能够客观反映发动机性 能衰退情况, 对发动机性能参数进行特征研究, 有助于分析发动机性能状态 $[3,4]$ 。

近些年来, 非线性科学取得了蓬勃发展. 因为她运用特殊的思想和方法研究自然界和人类社会, 解决和 说明了一些传统科学所不能解决的问题和现象, 其应用和研究已经渗透到了众多学科和领域。分形分析作 为非线性科学的分支, 自上世纪被引入后就引起了人们极大的兴趣。近些年来, 随着多重分形理论研究的 深入, 它已被成功的应用到物理学、气象学、经济学、生命科学等领域 [5-9]。本文尝试采用分形理论研究 航空发动机性能参数的非线性结构，探讨其在航空发动机健康监控中的应用。

本文的组织结构如下：第 1 部分描述本文用于分析的数据; 第 2 部分给出本文应用的方法以及应用数 据得到的分形特性; 第 3 部分是文章得出的重要结论。

\section{2 数据描述}

航空发动机性能可用多个参数来描述 [10]，如排气温度 (Teg)、燃油流量 (Wf)、转子转速 (N1 和 N2)、 
滑油压力 (Poi) 以及滑油温度 (Toi) 等。本文主要分析燃油流量时间序列的分形特征, 原始数据来自中国某 航空公司某型波音 777 客机 PW4077 发动机近一年的巡航飞行数据。

\section{3 方法论与结果}

\section{1 统计矩}

假设要研究的时间序列为 $\{X(u): u \in[0, U]\}$ 。首先把区间 $[0, U]$ 分割成若干不相交的小区间, 设 $\lambda$ 为 小区间长度与总区间长度比值的最大值, 记整个区间序列数量总和为 $\mathrm{M}$, 即

$$
\mathrm{M}=\sum_{u \in[0, \mathrm{U}]} \mathrm{X}(\mathrm{u})
$$

第 $i$ 个小区间概率密度分布函数

$$
p_{i}(\lambda)=\frac{N(\lambda, i)}{M}
$$

定义统计矩函数 (也称为配分函数) $\chi_{q}(\lambda)$ :

$$
\chi_{q}(\lambda) \equiv \sum_{i}\left(p_{i}(\lambda)\right)^{q}
$$

指数 $q$ 描述了统计矩的阶, 理论上是一个从 $-\infty$ 到 $+\infty$ 的数值, 并规定 $0^{0}=0$ 。

若统计矩服从幂律关系: $\chi_{q}(\lambda)=\sum\left(p_{i}(\lambda)\right)^{q} \propto \lambda^{\tau(q)}$, 则这个幕律关系通常反映在双对数关系图 $\log \chi_{q}(\lambda) \sim \log \lambda$ 上。在满足幂律关系的尺度区间, 我们可以通过双对数关系图来确定配分函数 $\tau(q)$,

$$
\tau(q)=\lim _{\lambda \rightarrow 0} \frac{\log \chi_{q}(\lambda)}{\log \lambda}
$$

通过分析 $\tau(q)$ 与 $q$ 的关系, 如果两者之间存在着非线性关系, 则说明序列具有多重分形性; 如果 $\tau(q)$ 是 $q$ 的线性函数, 则说明序列是单分形的。

图 1 显示了 $q \in(-50,50)$ 时配分函数的关系图, 可以看出 $\tau(q)$ 是一个非线性的函数, 即 $\tau(q)$ 与 $q$ 之 间存在着弱非线性关系，这也表明燃油流量时间序列的确具有弱重分形的特性。 


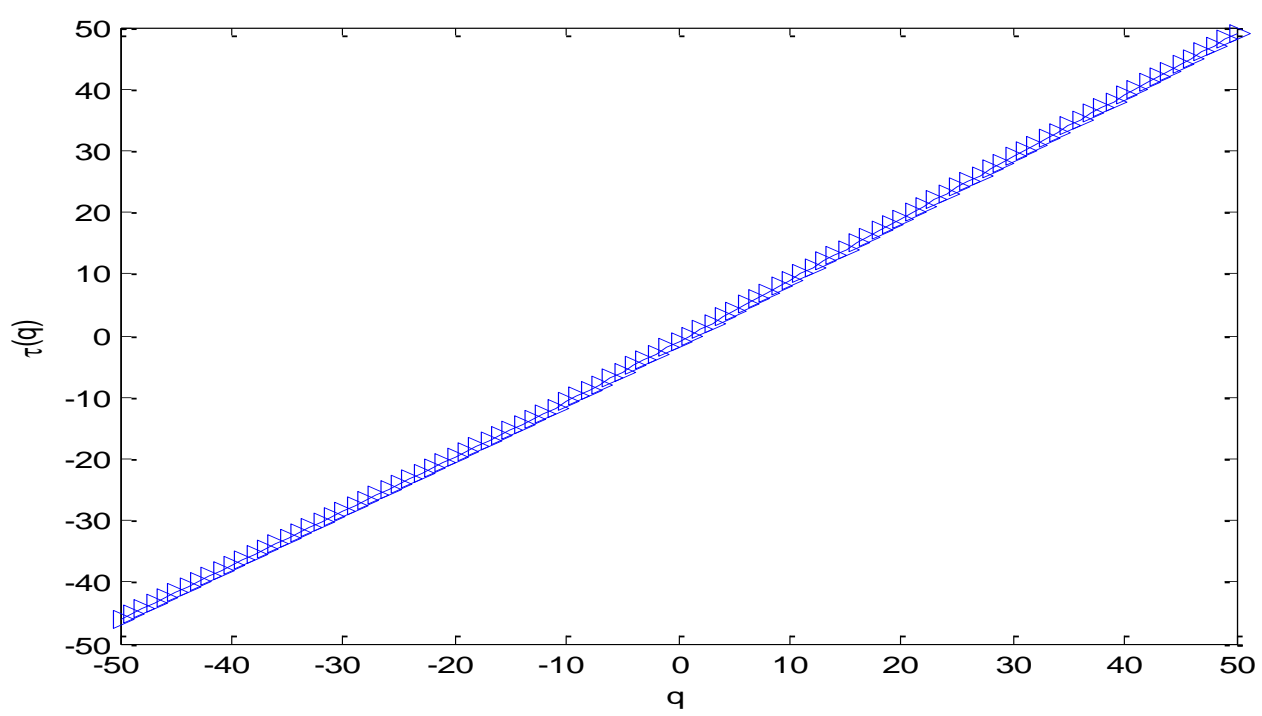

图 1 配分函数 $\tau(q) \sim q$

\section{2 广义分形维数}

广义分形维数 $D(q)$ 的定义为:

$$
D(q)=\left\{\begin{array}{l}
\frac{1}{q-1} \lim _{\lambda \rightarrow \infty} \frac{\log \chi_{q}(\lambda)}{\log \lambda}, \ldots \ldots q \neq 1 \\
\lim _{\lambda \rightarrow \infty} \frac{\sum_{i} p_{i}(\lambda) \log p_{i}(\lambda)}{\log \lambda}, \ldots \ldots q=1
\end{array}\right.
$$

它与配分函数 $\tau(q)$ 具有如下关系:

$$
D(q)=\left\{\begin{array}{l}
\frac{\tau(q)}{q-1}, \ldots . . q \neq 1 \\
\tau^{\prime}(1), \ldots . . q=1 \text { 且 } \tau(q) \text { 可微 }
\end{array}\right.
$$

特别地, 当 $q=1_{\text {时, }} D(1)$ 可以通过下式计算:

$$
D(1)=\lim _{\lambda \rightarrow 0} \frac{\sum_{i} p_{i}(\lambda) \log p_{i}(\lambda)}{\log \lambda}
$$

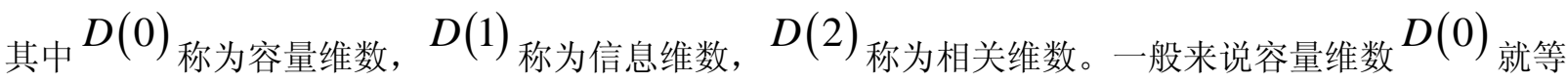
于多重分形谱的极大值。

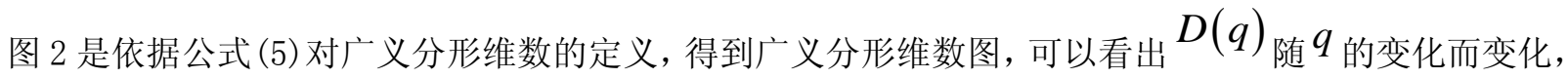
这也表明燃油流量时间序列具有多重分形特征。图中可以看出 $D(q) \in(0.9,1)$, 因此该序列具有较弱的多重 分形性。 


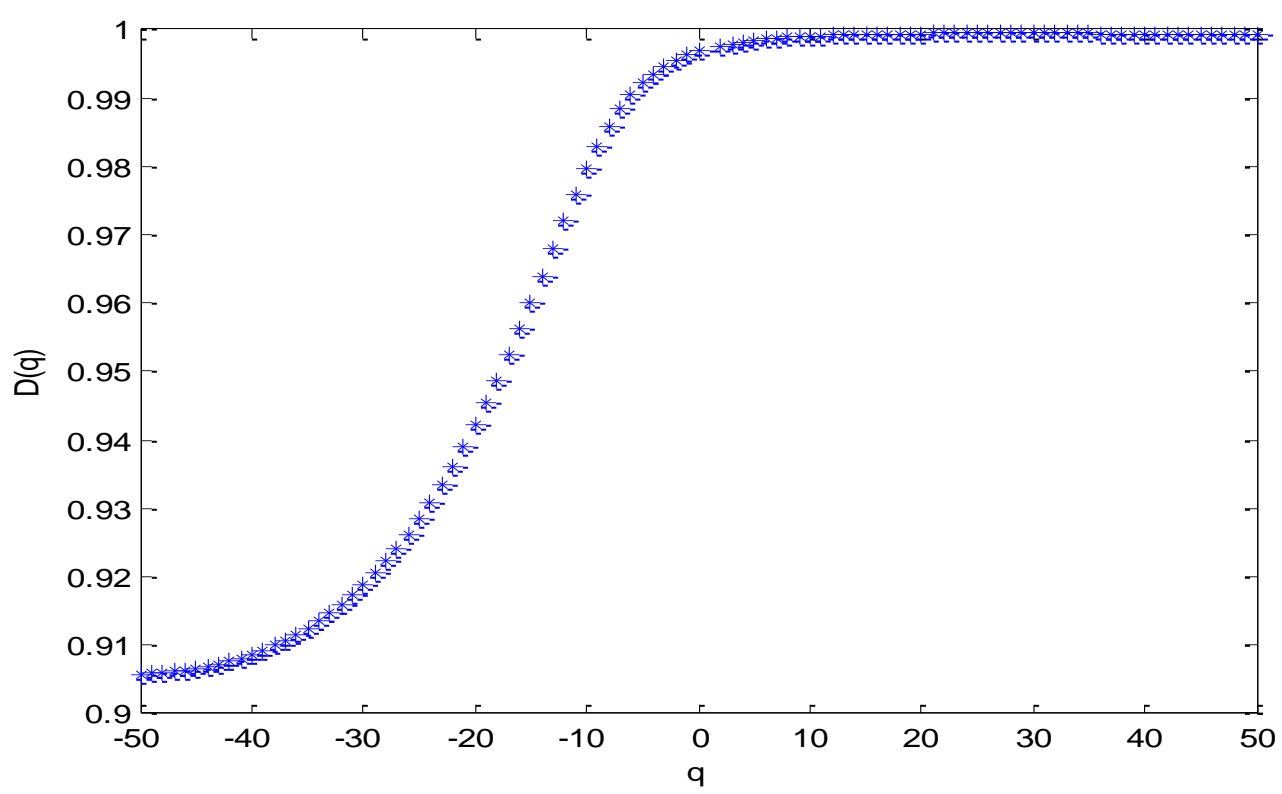

图 2 广义分形维数 $D(q) \sim q$

\section{4 结论}

本文简要介绍了配分函数和广义分形维数的概念，并从这两个角度对航空发动机燃油流量时间序列进 行了研究。首先, 配分函数显示了一定的非线性特征, 这表明燃油流量时间序列具有一定的多重分形性。 进一步地, 通过估算该序列广义分形维数, 证实了燃油流量时间序列具有较弱的多重分形性质。这一结果 将为航空发动机其它性能参数的分形特征研究提供参考。

\section{5 致谢}

感谢国家自然科学基金项目（批准号：61401467 ), 天津中德应用技术大学科技一般项目（批准 号:zdkt2015-006 ), 中国民航大学基本科研业务费(批准号：3122017084)资助。

\section{参考文献:}

[1] R. Ganguli, Application of Fuzzy Logic for Fault Isolation of Jet Engines, J. Eng. Gas. Turb. Power. 125 (2003) 617-623..

[2] Van der Weide J A M, Pandey M D, Van Noortwijk J M. Discounted cost model for condition based maintenance optimization[J]. Reliability Engineering and System Safety, 2010, 95(3): 236-246.

[3] K. Dong, J. Fan, Y. Gao, Cross-correlations and Structures of Aero-engine Gas Path System Based on DCCA Coefficient and Rooted Tree, Fluct. Noise Lett. 14 (2015) 1550014.

[4] K. Dong, Y. Gao, L. Jing, Correlation Tests of the Engine Performance Parameter by Using the Detrended Cross-correlation Coefficient, J. Korean Phys. Soc. 66 (2015) 539-5435.

[5] X. Zhao, P. Shang, W. Shi, Multifractal cross-correlation spectra analysis on Chinese stock markets, Physica A. 402 (2014) 89-92.

[6] E.B.S. Marinho, A.M.Y.R. Sousa, R.F.S. Andrade, Using detrended cross-correlation analysis in geophysical data, Physica A. 392 (2013) 2195-2201.

[7] Falconer KJ. Fractal Geometry-Mathematical Foundations and Applications. New York: John Wiley \& Sons(1990). 
[8] B S Kerner. The Physics of Traffic[M]. New York. Springer. 2004

[9] X. Zhao, P. Shang, A. Lin, G. Chen, Multifractal Fourier detrended cross-correlation analysis of traffic signals, Physica A. 390 (2011) 3670-3678.

[10]B. Zhou. Design and implementation of QAR data processing system [D].Tianjin: College of Aeronautical Engineering, Civil Aviation University of China, 2009. 